\title{
Antimicrobial activity of Salvadora persica on Streptococcus pneumoniae.
}

\author{
Mohammed K. Almaghrabi* \\ Department of Microbiology and Clinical Parasitology, College of Medicine, King Khalid University, Abha, Saudi \\ Arabia
}

\begin{abstract}
Streptococcus pneumoniae is an important pathogen, which is mainly the causative agent of pneumonia. The limited treatment and control options mean refers an urgent need for novel effective therapies to treat infected patients. The study has described one approach of controlling and treating this pathogen using Salvadora persica. An extract from different parts of this plant has been used including twigs, fruit, and root. All extracts were shown to clear pneumococci lawns on solid media. However, fruit extract showed a considered reduction in pneumococcal colony forming unit (CFU) in liquid culture. This represents a promising therapeutic strategy against pneumococcal infection in particular by highly invasive strains that have evaded vaccination, or are resistant to existing antibiotics.
\end{abstract}

Keywords: Antimicrobial, Salvadora persica, Streptococcus pneumonia, Fruit extract, Resistance, Antibiotics. Accepted on October 22, 2018

\section{Introduction}

Streptococcus pneumoniae is a major cause of pneumonia, sepsis, and meningitis, responsible for over 1.2 million deaths per year. Worldwide, pneumonia is the leading infectious cause of mortality among children and adults, with Streptococcus pneumoniae being the most commonly recovered isolate [1]. In developing countries, respiratory tract infections frequently progress to fatal sepsis and meningitis, particularly in the immunocompromised, young, and elderly [2]. Approximately $50 \%$ of deaths occur during the first $48 \mathrm{~h}$ of treatment, where antibiotic therapy affects mortality during this period [3-5]. Furthermore, rates of antibiotic resistance are rising with over one third of isolates in the US and parts of Europe showing reduced susceptibility to penicillin [6,7]. As, pneumococcal conjugate vaccines have resulted in a decline in invasive disease caused by pneumococcal serotypes included in the vaccine [8], non-vaccine serotypes have been shown to cause replacement disease. Another antimicrobial agent should be discovered to be used against this bacterium to address these important clinical challenges and provide cross serotype protection against pneumococcal infection. One such source of a new antimicrobial; is a Salvadora persica.

S. persica, locally called miswak is an ancient toothbrush belongs to the Salvadoraceae family. It is scientifically proven to prevent dental decay when it is used for tooth cleaning [9]. Different parts of this plant such as root, twigs, and stem are used in Middle East to obtain dental hygiene. The use of high concentration of $S$. perscia extract gave similar activity to that obtained by using oral disinfectants and anti-plaque agents, such as triclosan and chlorhexidine gluconate [10,11]. Studies have shown that miswak extract contains antibacterial, antifungal and anti-plasmodial effects [12-14]. The present study has investigated the impact of $S$. persica root, fruit, and twig extract on the most common causative communityacquired pneumonia, Streptococcus pneumoniae.

\section{Materials and Methods}

\section{Plant materials}

Fresh and healthy root, fruit, and twigs of the plant $S$. persica were collected from local farms in Hali centre, Alqunfudah city, province of Makkah, Saudi Arabia (Figure 1). The collection of the plant component was undertaken in June 2014.

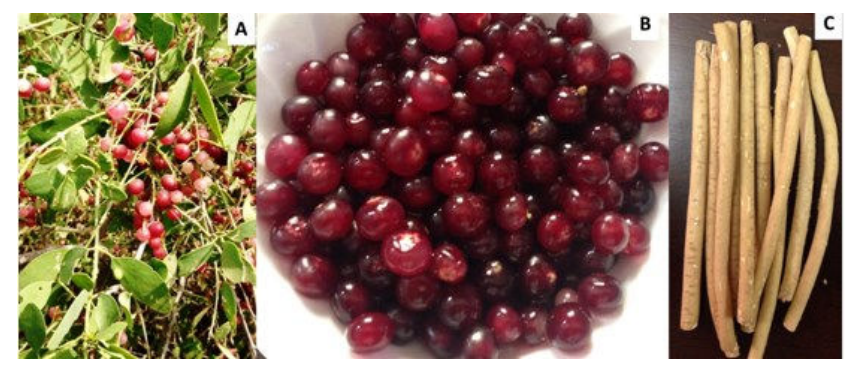

Figure 1. Salvadora persica tree; A: Twigs with fruits, B: Fruit and C: root.

\section{Preparation of plant materials}

Roots, twigs, and fruit of S. persica were washed several times with water and then were left to dry in air. A $500 \mathrm{~g}$ of root and twigs were grinded and homogenized with $250 \mu \mathrm{l}$ of water. Fruits were directly homogenized without the addition of 
water. All different aqueous extracts were centrifuged and then filtered with $022 \mu \mathrm{m}$ pore size filters.

\section{Bacterial strain}

Laboratory strain (D39) of Streptococcus pneumoniae was used in this study. The bacteria strain was provided from laboratory beads collection stocks of Professor Aras Kadioglu, University of Liverpool, United Kingdom.

\section{Culture media}

Blood agar (Oxoid, UK) was prepared according to the manufacturer's instruction, autoclaved, cooled down, and 5\% of horse blood was added. Medium then was dispensed at 20 $\mathrm{ml}$ per plate in $12 \times 12 \mathrm{~cm}$ Petri dishes. Set plates were incubated overnight to ensure sterility before use.

\section{Antimicrobial activity}

Determination of zone of inhibition method: Antimicrobial activity was undertaken using spot test as described by Armon and Kott [15]. $500 \mu \mathrm{l}$ of exponential growth of strain D39 was added to $3 \mathrm{ml}$ of $0.4 \%$ sloppy agar (3.7 $\mathrm{g}$ brain heart infusion (BHI) broth base and $0.4 \mathrm{~g}$ of agar). The mixture was then poured on BHI agar base, distributed all over the plate, and left on the bench to set. 10 to $20 \mu \mathrm{l}$ of extract was spotted on the top of pneumococcal lawns, once lawns were made and the agar set. Spotted plates were left on the bench to dry and then incubated overnight at $37^{\circ} \mathrm{C}$ using an-aerobic conditions. The plates were observed the next day to monitor the presence of zone of killing, which indicate the antimicrobial activity.

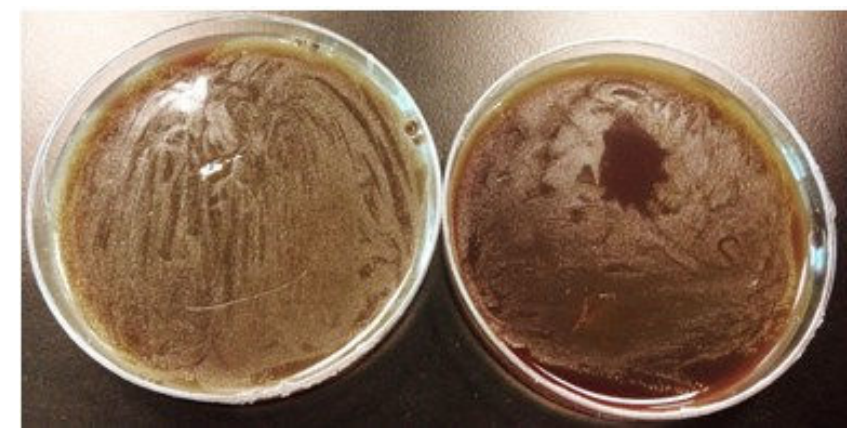

Figure 2. Antimicrobial activity of Salvadora persica extracts on pneumococcal lawn (Right plate is a control and left plate tested culture which shown a clear zone of inhibition).

Antimicrobial effect on bacterial growth: Colony-forming units were enumerated using the Miles-Misra assay with some modifications to evaluate the effect of $S$. persica extract on pneumococcal growth. Triplicate dilutions of $10^{-1}$ of each plant extracts were made using BHI-serum broth $(20 \%$ of foetal calf serum) and were kept for further processes. Tube of $10 \mathrm{ml}$ of brain heart infusion (BHI) broth was inoculated with strain D39 and incubated overnight at $37^{\circ} \mathrm{C}$. The following day, they were centrifuged at $1750 \mathrm{Xg}$ for $15 \mathrm{~min}$. The pellets were resuspended in $1 \mathrm{ml}$ of extract/BHI-serum broth, which were prepared earlier. $700 \mu \mathrm{l}$ from the re-suspended pellets for each serotype was added to $10 \mathrm{ml}$ of BHI-serum broth, which was then incubated at $37^{\circ} \mathrm{C}$. The blood agar plate was divided into six sections. Three drops of $20 \mu \mathrm{l}$ of each culture were spotted over each section. The previous process was assessed on two plates and repeated twice. All plates were incubated in $\mathrm{CO}_{2}$ gas jar at $37^{\circ} \mathrm{C}$ overnight. The following day, the dilution containing the optimum numbers of colonies ( 200 colonies) was counted and calculated the viable count using the following formula;

CFU per $\mathrm{ml}=$ Mean number of colonies in sector $\times$ Dilution $\times$ $1000 / 60$.

\section{Results and Discussion}

\section{Determination of zone of inhibition method}

A zone of killing on spotted areas has been observed (Figure 2 ). The zone of bacterial growth inhibition was very clear using all extract indicating that $S$. persica extracts have a bactericidal effect on $S$. pneumoniae growth.

\section{Antimicrobial effect of $S$ persica extracts on pneumococcal growth in liquid culture}

The effect of $S$. persica extracts on pneumococcal growth in liquid culture indicated that only fruit extract has shown the ability to suppress pneumococcal growth. The initial CFU counts of treated cultures were $10^{7}$ and it continued at the same value using fruit extract for five hours. At the end of the experiment, fruit extract-treated culture CFU was approximately $1.25 \log \mathrm{CFU}$ lower than control. Whereas, viable count of those cultures which treated with root and twigs extract was only $0.1 \mathrm{log}$ CFU lower than control (Figure 3). Fruit treated cultures have shown approximately $1.25 \log$ CFU difference as compared to control; whereas, root and twigs treated cultures had no significant efficacy on pneumococcal CFUs.

\section{The effect of $S$. persica extract on S. pneumoniae growth}

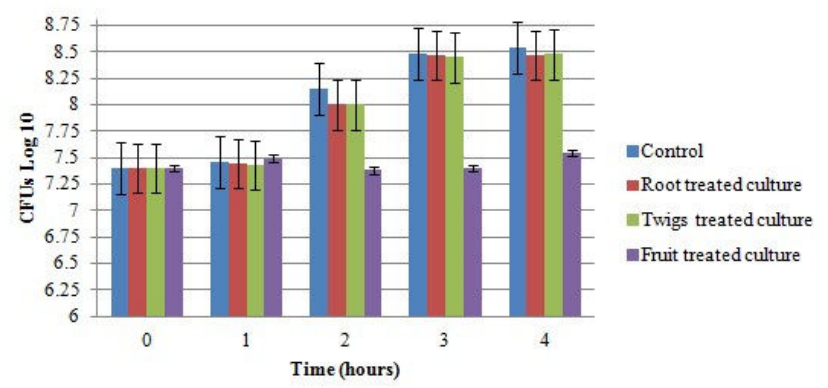

Figure 3. Effect of S. persica extracts on pneumococcal growth in liquid culture.

Medicinal plant extracts are being increasingly reported as antimicrobial agents from different parts of the world. The World Health Organization (WHO) has estimated that the extraction or the active constituents of such plant are used as traditional medicine in $80 \%$ of the world's population [16]. Ancient Arabs were familiar to Miswak to clean their teeth and 
get them white and shiny [15]. The same method of teeth cleaning approach was used in Japan and USA with different type of plants [17]. The efficacy of Miswak on different bacterial growth was extensively studied; including, Staphylococcus aureus [12], Streptococcus fecalis and S.mutans [18], Candida albicans [19], and Candida species [13].

In the present study, the extracts obtained from fruits, twigs, and root of Salvadora persica showed strong activity against $S$. pneumoniae wild type strains. The current results of this study might justify the ancient use of root and twigs of this plant as tooth brush. Zone of growth inhibition on pneumococcal lawn was observed by using extracts from twigs, fruits, and roots of $S$. persica that indicated their ability to kill pneumococcal cells. The effect of these extract on pneumococcal growth in liquid culture showed only fruit extract reduced pneumococcal CFUs in about one log difference as compared to controls. However, very small difference in log CFUs difference was obtained by using twigs and root extracts. The difference between the effect of twigs and root extract to clear pneumococci in lawn and broth might refer to the concentration of active material. Diluting the extracts during measuring their effect on pneumococcal in broth cultures might decreases the effect of antimicrobial agents indicating that the concentration of antimicrobial agent in fruit extract was higher than those in twigs and root extract.

\section{Acknowledgement}

The author is very thankful to all the associated personnel in any reference that contributed in/for the purpose of this research. Further, this research holds no conflict of interest and is not funded through any source.

\section{References}

1. Black RE, Cousens S, Johnson HL, Lawn JE, Rudan I, Bassani DG, Eisele T. Global, regional, and national causes of child mortality in 2008: a systematic analysis. Lancet 2010; 375: 1969-1987.

2. Gordon SB, Chaponda M, Walsh AL, Whitty CJ, Gordon MA, Machili CE, Molyneux ME. Pneumococcal disease in HIV-infected Malawian adults: acute mortality and long-term survival. AIDS (London, England) 2002; 16: 1409.

3. Imran MN, Leng PH, Yang S, Kurup A, Eng P. Early predictors of mortality in pneumococcal bacteraemia. Ann Acad Med Singapore 2005; 34: 426-431.

4. Mortensen EM, Restrepo MI, Anzueto A, Pugh JA. Antibiotic therapy and 48-hour mortality for patients with pneumonia. Am J Med 2006; 119: 859-864.

5. Tunkel AR. Adjunctive corticosteroids for adults in subSaharan Africa with bacterial meningitis. Curr Infect Dis Rep 2008; 10: 290-291.

6. Schito GC, Debbia EA, Marchese A. The evolving threat of antibiotic resistance in Europe: new data from the
Alexander Project. J Antimicrob Chemother 2000; 46: 3-9.

7. Thornsberry C, Ogilvie P, Kahn J, Mauriz Y. Surveillance of antimicrobial resistance in Streptococcus pneumoniae, Haemophilus influenzae, and Moraxella catarrhalis in the United States in 1996-1997 respiratory season. Diagn Microbiol Infect Dis 1997; 29: 249-257.

8. Pilishvili T, Lexau C, Farley MM, Hadler J, Harrison LH, Bennett NM, Smith PJ. Sustained reductions in invasive pneumococcal disease in the era of conjugate vaccine. J Infect Dis 2010; 201: 32-41.

9. Salehi P, Sh MD. Comparison of the antibacterial effects of persica mouthwash with chlorhexidine on streptococcus mutans in orthodontic patients. DARU J Pharm Sci 2006; 14: 178-182.

10. Almas K. The effect of Salvadora persica extract (miswak) and chlorhexidine gluconate on human dentin: a SEM study. J Contemp Dent Pract 2002; 3: 27-35.

11. Almas K, Skaug N, Ahmad I. An in vitro antimicrobial comparison of miswak extract with commercially available non-alcohol mouthrinses. Int J Dent Hyg 2005; 3: 18-24.

12. Al lafi $\mathrm{T}$, Ababneh $\mathrm{H}$. The effect of the extract of the miswak (chewing sticks) used in Jordan and the Middle East on oral bacteria. Int Dent J 1995; 45: 218-222.

13. al-Bagieh NH, Idowu A, Salako NO. Effect of aqueous extract of miswak on the in vitro growth of Candida albicans. Microbios 1994; 80: 107-113.

14. Ali H, Konig GM, Khalid SA, Wright AD, Kaminsky R. Evaluation of selected Sudanese medicinal plants for their in vitro activity against hemoflagellates, selected bacteria, HIV-1-RT and tyrosine kinase inhibitory, and for cytotoxicity. J Ethnopharmacol 2002; 83: 219-228.

15. Bos G. The miswãk, an aspect of dental care in Islam. Med Hist 1993; 37: 68-79.

16. Bhalodia NR, Shukla VJ. Antibacterial and antifungal activities from leaf extracts of Cassia fistula 1.: An ethnomedicinal plant. J Adv Pharm Technol Res 2011; 2: 104.

17. Hyson JM. History of the toothbrush. J Hist Dent 2003; 51: 73-80.

18. Almas K, Al-Bagieh NH. The antimicrobial effects of bark and pulp extracts of miswak, Salvadora persica. BML 1999; 60: 71-75.

19. Almas K, Stakiw JE. The effect of miswak extract from Salvadora persica stored for 18 years on microbes in vitro. Egypt Dent J 2000; 46: 227-230.

\section{*Correspondence to}

Mohammed K. Almaghrabi

Department of Microbiology and Clinical Parasitology

College of Medicine

King Khalid University

Saudi Arabia 